\title{
Slip Modeling and Estimation for a Planetary Exploration Rover: Experimental Results from Mt. Etna
}

\author{
Kristin Bussmann, Lukas Meyer, Florian Steidle, Armin Wedler
}

\begin{abstract}
For wheeled mobile systems, the wheel odometry is an important source of information about the current motion of the vehicle. It is used e.g. in the context of pose estimation and self-localization of planetary rovers, which is a crucial part of the success of planetary exploration missions. Depending on the wheel-soil interaction properties, wheel odometry measurements are subject to inherent errors such as wheel slippage. In this paper, a parameter-based approach for wholebody slip modeling and calibration is applied to a four-wheeled lightweight rover system. Details on the method for slip parameter calibration as well as the system-specific implementation are given. Experimental results from a test campaign on Mt. Etna are presented, showing significant improvements of the resulting wheel odometry measurements. The results are validated during a long range drive of approx. $900 \mathrm{~m}$ and discussed w. r.t. the advantages but also limitations of the method within a space exploration scenario.
\end{abstract}

\section{INTRODUCTION}

Due to the technological progress of the last decades, space exploration has become more than pure astrological observation. As the deployment of humans on foreign planets is complicated, dangerous and expensive, one key element for extraterrestrial on-surface exploration is the utilization of autonomous ground vehicles. The design of these planetary rovers does usually focus on robust locomotion on rough terrain, but must also feature the transportation of scientific payloads [1], [2].

The Lightweight Rover Unit (LRU) is a research prototype of a planetary exploration rover, built at the Robotics and Mechatronics Center of the German Aerospace Center according to space concept studies [3], [4]. The four independently steerable wheels as well as the active suspension system between the body and front and rear bogie axles were designed to feature high mobility in rough terrain. The software framework running on the rover provides a great level of autonomy [5], which is one of the key requirements for planetary exploration [6]. A crucial factor for the success of autonomous operations in GPS-denied environments is precise self-localization. At the LRU, this is realized by fusing sensory inputs of inertial measurement unit (IMU), visual odometry (VO) and wheel odometry (WO) within an extended Kalman Filter (EKF) [3] based on the concept of [7], [8].

The two varieties of the LRU (LRU1 with a science camera, and LRU2 equipped with a robotic arm for manipulation

This work was supported by the Helmholtz Association, project alliance ROBEX, under contract number HA-304.

The authors are with the Institute of Robotics and Mechatronics, German Aerospace Center (DLR). Contact: kristin.bussmannedlr. de

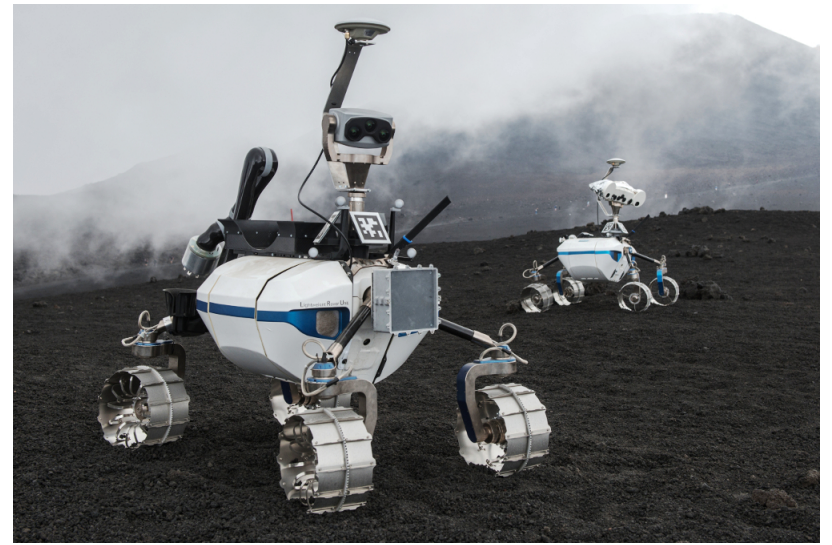

Fig. 1. The LRU rovers during the ROBEX Demo Mission Space at Mt Etna. Front: LRU2 with manipulator. Back: LRU1 with Science Camera.

purposes, see Fig. 1) served as robotic demonstrators during the ROBEX project alliance [9]. In this context a moon analogue demonstration mission was performed on Mt. Etna during June and July 2017 [10], putting into practice the experimental setup proposed by [11]. The main task of the mission was the autonomous deployment of seismometer boxes in a pre-defined layout in a previously unknown environment, requiring long-distance traverses. The volcanic soil as well as slopes on the test site lead to wheel slippage and thus posed big challenges to the rover's locomotion and self-localization capabilities. To improve the performance of the wheel odometry computation, a strategy for modeling and calibrating the overall slippage of the rover was needed.

There exist a multitude of approaches for this problem. One possibility is the physical modeling of wheel-soil contact of single wheels, e.g. [12] and a broad collection of references therein. These approaches usually make use of soil parameters, which cannot be assumed to be known a priori in unknown environments. Other works focus on the overall slippage of the vehicle. For example, [13] uses IMU and VO measurements as a reference to estimate WO errors. In this case, WO can be used for traction control, but cannot be fused with the reference signals to improve pose estimation due to correlations in the EKF. An estimation of all-wheel slippage based on measurements of the motor currents is performed in [14]. However, this approach does not give any information on lateral slip. Due to practical considerations - among others availability of ground truth measurements, computational feasibility, robustness in outdoor environments - we decided to adopt an approach that models all-wheel slip, based on the integrated prediction error minimization (IPEM) method by [15] and [16], and adjust it to the specific 


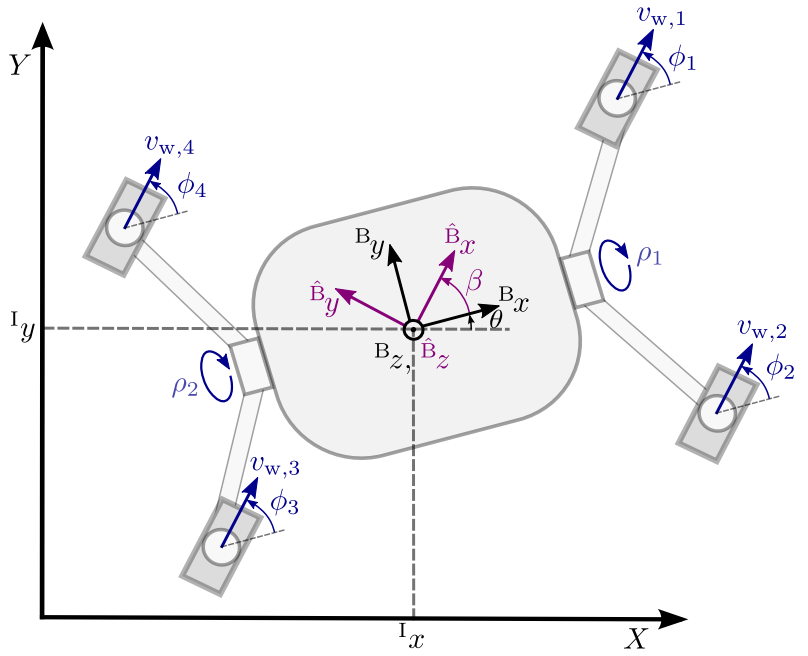

Fig. 2. Schematic drawing of the LRU in a plane. Wheel velocities are denoted by $v_{\mathrm{w}, 1 \ldots 4}$, steering angles by $\phi_{1 \ldots 4}$ and the front and rear angles between body and bogie axles by $\rho_{1 / 2}$. The body frame $\mathrm{B}$ is geometrically fixed at the center of the rover body. The drive frame $\hat{\mathrm{B}}$ is the rotated by $\rho_{1 / 2}$ and $\beta$ such that the momentary driving direction is the $x$-axis and the $z$-axis is vertical to the plane spanned by the wheels. The rover position in the inertial frame $(\mathrm{X}, \mathrm{Y})$ is denoted by the index $\mathrm{I}$.

use case. The greatest advantage of this algorithm is that no continuous ground-truth position or velocity measurements of the rover are needed, neither during the calibration phase nor during the autonomous operations of the space vehicle. This is a crucial requirement for the approach to work in the context of planetary exploration, where usually no such measurements can be provided.

In the course of this paper, the implementation and calibration of the slip model and the resulting slip-aware wheel odometry are described. In Sec. II, a brief introduction of the theoretical background and the method used for slip modeling and estimation is given. Thereby, specific solutions accounting for the rover design and the test site on Mt. Etna featuring volcanic soil and slopes up to $18 \mathrm{deg}$ are pointed out. Experimental results collected during the ROBEX demo mission on Mt. Etna are presented in Sec. III, including the calibration procedure and a long-range drive of approx. $900 \mathrm{~m}$. The application of the slip-aware wheel odometry within the pose estimation framework on the LRU is particularly considered during evaluation. The obtained results are discussed in the context of a planetary exploration scenario, the application specific challenges are outlined and coping strategies are provided (Sec. IV).

\section{Slip Model and Parameter Estimation}

A sketch of the rover with all relevant joint variables and frames can be inspected in Fig. 2. Note that the frames and their transformations are defined in 3D and are only depicted in $2 \mathrm{D}$ for the sake of clarity.

\section{A. Wheel Odometry and Slip Modeling}

The wheel odometry is computed by a kinematic model of the rover that maps the measured velocities of all four wheels to the center of the rover body while considering all relevant joint angles. The forward kinematics equation for one wheel $i \in\{1 \ldots 4\}$ reads

$$
{ }^{\mathrm{B}} \boldsymbol{v}_{\mathrm{w}, i}={ }^{\mathrm{B}} \boldsymbol{R}_{\mathrm{w}, i}\left(\phi_{i}, \rho_{j}\right)^{\mathrm{w}, i} \boldsymbol{v}_{\mathrm{w}, i}=\boldsymbol{J}_{\nu}\left(\rho_{j}\right)^{\mathrm{B}} \boldsymbol{\nu}-\boldsymbol{J}_{\rho}\left(\rho_{j}\right) \dot{\rho}_{j}
$$

where $\boldsymbol{v}_{\mathrm{w}, i} \in \mathbb{R}^{3}$ is the vector of translational velocities in the center of wheel $i$, expressed in body frame $\mathrm{B}$ or wheel frame $\mathrm{w}, i$. The frames can be switched by applying the rotation matrix ${ }^{\mathrm{B}} \boldsymbol{R}_{\mathrm{w}, i}$. The matrices $\boldsymbol{J}_{\nu} \in \mathbb{R}^{3 \times 6}$ and $\boldsymbol{J}_{\rho} \in \mathbb{R}^{3 \times 1}$ are the Jacobian matrices related to the spacial velocity of the rover body ${ }^{\mathrm{B}} \boldsymbol{\nu} \in \mathbb{R}^{6}$ and the bogie angular velocities $\dot{\rho}_{j}, j \in\{1,2\}$, respectively.

Equation (1) written for all four wheels can be stacked and solved for the body velocity ${ }^{\mathrm{B}} \boldsymbol{\nu}$ by a Moore-Penrose pseudoinverse of the stacked Jacobian matrix $\overline{\boldsymbol{J}}_{\nu} \in \mathbb{R}^{12 \times 6}$. This is analogue to [16] and provides the wheel odometry ${ }^{\mathrm{B}} \boldsymbol{\nu}_{\mathrm{WO}}=$ $\left({ }^{\mathrm{B}} \boldsymbol{v}_{\mathrm{WO}}^{T}{ }^{\mathrm{B}} \boldsymbol{\omega}_{\mathrm{WO}}^{T}\right)^{T}$. Therefore, the computation of the spatial body velocity of the rover depends on measurements of the wheel velocities along with additional rover telemetry data like steering and bogie angles. Linear and angular velocity components are denoted by $\boldsymbol{v}$ and $\boldsymbol{\omega}$, respectively.

Due to the fact that the wheels feature a low-level velocity controller that produces velocities consistent with the desired body velocity, we assume that slip occurs at all wheels simultaneously on loose soil. Therefore, slip can be modeled as error in the measured body velocity provided by the wheel odometry [15], thus resulting in the corrected velocity

$$
{ }^{\mathrm{B}} \hat{\boldsymbol{\nu}}_{\mathrm{WO}}={ }^{\mathrm{B}} \boldsymbol{\nu}_{\mathrm{WO}}-{ }^{\mathrm{B}} \boldsymbol{\nu}_{\mathrm{slip}}\left(\boldsymbol{p}, \boldsymbol{\nu}_{\mathrm{WO}}, \boldsymbol{F}\right) \text {. }
$$

There, the vector $\boldsymbol{p} \in \mathbb{R}^{m}$ contains $m$ slip parameters depending on the choice of the slip model - and $\boldsymbol{F} \in \mathbb{R}^{3}$ denotes the linear forces acting on the rover. A possible choice for a slip model is the linear six-parameter model proposed by [16] which will be detailed in Sec. II-C.

The crucial task for slip estimation is defining the parameter set $\boldsymbol{p}$ for the current soil. A general, non-linear model

$$
\begin{aligned}
\dot{\boldsymbol{\zeta}}(t) & =\boldsymbol{f}(\boldsymbol{\zeta}(t), \boldsymbol{u}(\boldsymbol{p}, t)), \\
\boldsymbol{\xi}(t) & =\boldsymbol{h}(\boldsymbol{\zeta}(t)) .
\end{aligned}
$$

is considered. In our case, the system state $\zeta \in \mathbb{R}^{6}$ is the rover pose expressed in an inertial frame I and $\boldsymbol{\xi}$ denotes the output measurement, that is the measured pose $\boldsymbol{\zeta}\left(t_{\text {end }}\right)$ at the end of a driven trajectory. The input vector $\boldsymbol{u}$ represents the body velocity ${ }^{\mathrm{B}} \boldsymbol{\nu}$.

Using system identification techniques, the parameters $\boldsymbol{p}$ can be determined such that modeling errors are minimized.

\section{B. Slip Parameter Calibration with IPEM}

Most methods to find $\boldsymbol{p}$ require measurements of the rover pose for all times $t \in\left[t_{0}, t_{\text {end }}\right]$ of a trajectory. However, the main advantage of the IPEM approach introduced by [15] is that no continuous information on the real trajectory is necessary. In the following section, the theoretical background of IPEM is recapitulated from [15].

The parameter set $\boldsymbol{p}$ can be identified by comparing the estimated (predicted) output of the system model with the measured output of the real system at the end of a driven 
trajectory with known start point. Additionally, continuous measurements of the system inputs $\boldsymbol{u}$ need to be recorded.

The dynamic equations (3) cover a whole trajectory and can be written in integral form as

$$
\begin{aligned}
\boldsymbol{\zeta}\left(t_{\mathrm{end}}\right) & =\boldsymbol{\zeta}\left(t_{0}\right)+\int_{t_{0}}^{t_{\mathrm{end}}} \boldsymbol{f}(\boldsymbol{\zeta}(\tau), \boldsymbol{u}(\boldsymbol{p}, \tau)) \mathrm{d} \tau \\
& =\boldsymbol{g}\left(\boldsymbol{\zeta}\left(t_{0}\right), \boldsymbol{u}(\boldsymbol{p}, . .)\right)
\end{aligned}
$$

where $\boldsymbol{u}(\boldsymbol{p}, .$.$) denotes the combined system input for all \tau \in$ $\left[t_{0}, t_{\text {end }}\right]$. The system output is defined as the measurement of the end point of a trajectory:

$$
\boldsymbol{\xi}_{\mathrm{msr}}=\boldsymbol{\zeta}_{\mathrm{msr}}\left(t_{\mathrm{end}}\right) \text {. }
$$

The estimated system output depends on the parameters $\boldsymbol{p}$ and is written as

$$
\boldsymbol{\xi}_{\text {est }}=\boldsymbol{h}(\boldsymbol{p})=\boldsymbol{\zeta}_{\text {pred }}\left(t_{\text {end }}\right) .
$$

Considering the error $\tilde{\xi}$ between the estimated and the real output, the following relation holds:

$$
\tilde{\boldsymbol{\xi}}=\boldsymbol{\xi}_{\mathrm{msr}}-\boldsymbol{h}(\boldsymbol{p})=\boldsymbol{H}_{\mathrm{sys}} \Delta \boldsymbol{p} .
$$

where $\Delta \boldsymbol{p}$ is a change in parameters that influences the estimation error $\tilde{\boldsymbol{\xi}}$. The matrix $\boldsymbol{H}_{\text {sys }}$ is called the parameter Jacobian matrix of a trajectory and models the influence that each parameter has on the estimated end point of the corresponding trajectory. This relationship is usually nonlinear and the parameter Jacobian matrix is the linearized representation:

$$
\boldsymbol{H}_{\mathrm{sys}}=\left[\frac{\partial \boldsymbol{g}}{\partial p_{1}}, \ldots, \frac{\partial \boldsymbol{g}}{\partial p_{m}}\right] .
$$

To avoid parameter overfitting and keep the influence of measurement noise as small as possible, it is beneficial to render equation (7) overdetermined. For the determination of $m$ slip parameters, this is achieved by recording a set of $n$ driven trajectories with $n \cdot \operatorname{dim}(\boldsymbol{\xi}) \gg m$.

The respective parameter Jacobian matrices are stacked, and (7) yields an over-constrained system, which is solved using the generalized inverse of the stacked parameter Jacobian matrices with the positive definite weighting matrix $\boldsymbol{W}$ :

$$
\Delta \boldsymbol{p}=\left[\begin{array}{c}
\boldsymbol{H}_{\mathrm{sys}}^{1} \\
\vdots \\
\boldsymbol{H}_{\mathrm{sys}}^{n}
\end{array}\right]^{\boldsymbol{W}+}\left[\begin{array}{c}
\boldsymbol{\xi}_{\mathrm{msr}}^{1}-\boldsymbol{h}(\boldsymbol{p})^{1} \\
\vdots \\
\boldsymbol{\xi}_{\mathrm{msr}}^{n}-\boldsymbol{h}(\boldsymbol{p})^{n}
\end{array}\right]
$$

The weighting matrix can be used e.g. in case the measurements are obtained using different methods or with different accuracy.

The parameter set $\boldsymbol{p}$ is then determined iteratively over several update steps $k$ with

$$
\boldsymbol{p}_{k+1}=\boldsymbol{p}_{k}+\alpha \Delta \boldsymbol{p}_{k}
$$

where $\alpha \in] 0,1]$ denotes the update rate.

For the parameter Jacobian matrix, an analytical solution can be found if simplifications are applied. These simplifications include the linearization of (3) and the use of the
2D pose for $\zeta$, solely considering the $x, y$ positions and the yaw angle $\theta$. This approach requires measurements in an inclined 2D plane as reference to consider slope dependent slip. Note that driving can be done in arbitrary terrain, only the measurements need to be done in the 2D plane - thanks to the endpoint-only dependency of IPEM. This approach has several advantages as it allows for reduced computational time and to use very simple measurement methods for the rover pose, while still providing good results [16].

Linearizing the system (3) and using the general solution for linear systems allows to rewrite (4) as [17]

$$
\begin{aligned}
& \boldsymbol{\zeta}\left(t_{\mathrm{end}}\right)=\boldsymbol{g}\left(\boldsymbol{\zeta}\left(t_{0}\right), \boldsymbol{u}(\boldsymbol{p}, . .)\right)= \\
& =\boldsymbol{\Phi}\left(t_{\mathrm{end}}, t_{0}\right) \boldsymbol{\zeta}\left(t_{0}\right)+\int_{t_{0}}^{t_{\mathrm{end}}} \boldsymbol{\Phi}\left(t_{\mathrm{end}}, \tau\right) \boldsymbol{G}(\tau) \boldsymbol{u}(\boldsymbol{p}, \tau) \mathrm{d} \tau
\end{aligned}
$$

The transition matrix

$$
\boldsymbol{\Phi}(t, \tau)=\left[\begin{array}{ccc}
1 & 0 & -\left({ }^{I} y(t)-{ }^{I} y(\tau)\right) \\
0 & 1 & { }^{I} x(t)-{ }^{I} x(\tau) \\
0 & 0 & 1
\end{array}\right]
$$

thereby models the propagation of the wheel odometry error over the time and its impact on the pose. See [17] for details on the derivation of $\boldsymbol{\Phi}$ for wheeled robots. The input body velocities $\boldsymbol{u}$ are transformed into the inertial frame by $\boldsymbol{G}$ with

$$
\boldsymbol{G}=\left[\begin{array}{ccc}
\cos (\theta) & -\sin (\theta) & 0 \\
\sin (\theta) & \cos (\theta) & 0 \\
0 & 0 & 1
\end{array}\right]
$$

for the two dimensional case. The pose (11) at the end of the trajectory is inserted into (8) and the derivative is moved inside the integral. The resulting expression for the parameter Jacobian matrix can therefore be written as

$$
\boldsymbol{H}_{\mathrm{sys}} \approx \int_{t_{0}}^{t_{\mathrm{end}}} \boldsymbol{\Phi}\left(t_{\mathrm{end}}, \tau\right) \boldsymbol{G}(\tau) \frac{\partial \boldsymbol{u}(\boldsymbol{p}, \tau)}{\partial \boldsymbol{p}} \mathrm{d} \tau .
$$

Another possibility for the determination of $\boldsymbol{H}_{\text {sys }}$ is the computation of the numerical derivative according to (8). This allows to consider the full 6DOF of the rover pose and yields the most precise results at the cost of increased computational requirements, but can also lead to significant numerical errors.

Using the analytical solution for the parameter Jacobian matrix is necessary in applications where fast computation is required, for example online parameter estimation. Furthermore, there is no advantage in computing the 6DOF $\boldsymbol{H}_{\text {sys }}$ in cases where no full 6DOF measurements of the rover pose are available.

During the ROBEX mission, the IPEM approach recalled from [15] was used to calibrate the slip-aware wheel odometry on the LRU. System-specific implementation details are given in the following.

\section{Implementation at the LRU}

For the application on the volcanic soil of Mt. Etna, we compared different parameterizations of the velocity error ${ }^{\mathrm{B}} \boldsymbol{\nu}_{\text {slip. }}$. In the end, it turned out that the best results were 


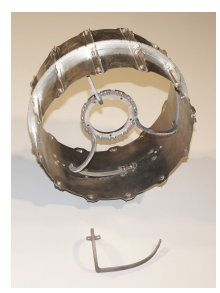

(a)

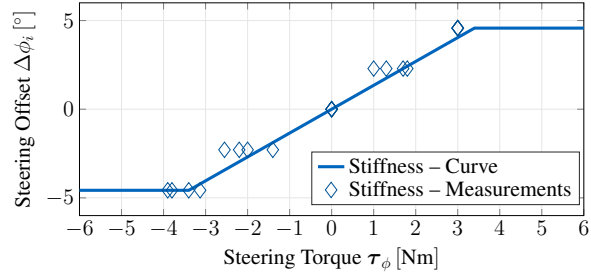

(b)
Fig. 3. (a) LRU wheel with three laster-sintered titan spokes (top) and single titan spoke (bottom) [5]. (b) Saturated spring characteristics for the steering actuators of the LRU. The averaged slope is $1.35 \frac{\mathrm{deg}}{\mathrm{Nm}}$.

achieved by the six-parameter slip-velocity model of [16], where the linear and angular velocity error components are calculated as

$$
{ }^{\hat{\mathrm{B}}} \boldsymbol{v}_{\text {slip }}=\left(p_{1} \frac{{ }^{\hat{\mathrm{B}}} F_{x}}{\hat{\mathrm{B}} F_{z}}{ }^{\hat{\mathrm{B}}} v_{x}+p_{2}{ }^{\hat{\mathrm{B}}} v_{x}\right) \hat{\mathrm{B}} \boldsymbol{e}_{x}+p_{3} \frac{\hat{\mathrm{B}} F_{y}}{{ }_{\mathrm{B}}^{\mathrm{B}} F_{z}} v_{x}{ }^{\hat{\mathrm{B}}} \boldsymbol{e}_{y} \text {, }
$$

and

$$
{ }^{\hat{\mathrm{B}}} \boldsymbol{\omega}_{\text {slip }}=\left(p_{4} \frac{\hat{\mathrm{B}} F_{y}}{{ }_{\mathrm{B}}^{\mathrm{B}} F_{z}}{ }^{\hat{\mathrm{B}}} v_{x}+p_{5}{ }^{\hat{\mathrm{B}}} v_{x}+p_{6}{ }^{\hat{\mathrm{B}}} \omega_{z}\right){ }^{\hat{\mathrm{B}}} \boldsymbol{e}_{z},
$$

respectively. Thereby, $e \in \mathbb{R}^{3}$ is the unit vector. The indices $x, y$ and $z$ denote individual components of velocity, force, and parameter vectors in the drive frame $\hat{\mathrm{B}}$, that accounts for the instantaneous driving direction of the rover (cf. Fig. 2). The transformation into the drive frame consists of a rotation of the body frame $\mathrm{B}$ around the $x$ axis into the plane spanned by the wheels ${ }^{1}$ with the corresponding rotation matrix $\boldsymbol{R}_{x}(\rho)$. Afterwards, the resulting intermediate frame $\overline{\mathrm{B}}$ is rotated around the $z$ axis by an angle $\beta$, which computes to $\beta=\operatorname{atan} 2\left({ }^{\overline{\mathrm{B}}} v_{y},{ }^{\overline{\mathrm{B}}} v_{x}\right)$.

The first two parameters in $\boldsymbol{p}$ in (15) describe the slip in driving direction. Slip is slope dependent due to the decreased normal contact forces between wheels and the ground for increased inclinations. This slope-dependency is modeled by $p_{1}$. However, slip also has a slope-independent component due to the plastic deformation of the loose soil. In that case, wheels experience sinkage which causes slip due to both compaction resistance and bulldozing resistance [18] that can be combined into rolling resistance modeled by $p_{2}$. Side slip while traversing slopes is modeled by $p_{3}$ and depends on the inclination of the terrain, similar to the first parameter. The remaining parameters describe errors in angular velocity due to over- and under-steering $\left(p_{4}\right)$, wheel asymmetry $\left(p_{5}\right)$, and skidding $\left(p_{6}\right)$ [16]. Note that with $\boldsymbol{p}=$ const., this method makes the assumption of homogeneous soil and cannot be used in strongly varying terrain (e.g. mixture of rocky to sandy soils).

Experimental comparison showed that alternative slip parameter models (e.g. the additional consideration of acceleration, directed slope dependency - uphill and downhill slip modeled differently, or the 15-parameter approach from [15]) did not improve the results significantly but required

\footnotetext{
${ }^{1}$ during operation on Etna, the body was controlled such that $\rho_{1}=\rho_{2}$ and thus the four wheels spanned a plane.
}

a higher number of parameters, which therefore implies a greater number of required calibration trajectories. Thus, the presented six parameter model is considered as a trade-off between high modeling accuracy and low computational and experimental effort.

Concerning the computation of the parameter Jacobian matrix, the analytical solution (14) was chosen for the experiments with the LRU. First, it was intended to use the most straightforward way of determining the rover pose, that is in 2D (see Sec. III). Second, this choice allows to straightforwardly implement online parameter estimation, as discussed in [15]. There, an EKF is used to estimate $p$ while driving by considering piecewise sections of the drive, comparing it to directly available ground truth measurements. This approach is performed offline with the recorded data and results are shown in Sec. III. The online implementation is a topic of future work.

Eventually, another relevant effect can be observed during operation in rough terrain that is not modeled by (15). This is the systematic error between commanded and measured steering angles due to mechanical compliance of the wheels. The LRU wheels are equipped with three laser-sintered titan spokes respectively (cf. Fig. 3 (a)), which leads to mechanical flexibility of the rolling surface. On soft terrain, this ensures reduction of sinkage and, in combination with the grousers, gives the wheels maximum grip [5]. Additionally, torsional compliance between wheel hub and lateral surface is introduced by the wheel design. During the process of steering in loose soil, the wheels need to work against the terrain and need to push it aside. The resulting forces deflect the wheels and cause an error in the effective steering angle. The error can be modeled by considering the wheels as rotational springs and measuring the torques at the steering motors while using a saturated steering characteristic as depicted in Fig. 3 (b).

\section{EXPERIMENTS}

The following results were produced with the LRU1 rover on the test site Mt. Etna that is considered to be moonanalogue [19]. The experiments took place west of the Cisternazza crater, on relatively new volcanic soil from 2001 [20] that features rough, sharp-edged gravel with a particle size of around $0.5-1 \mathrm{~cm}$ diameter.

The first step was to calibrate the slip model as outlined in Sec. II. The procedure and results are described in Sec. IIIA. The obtained parameters were then used to improve the wheel odometry measurements during all other experiments of the entire mission. The performance was validated particularly during a long range run, corresponding results are shown in Sec. III-B. An impression of the experiments on Mt. Etna including the mission scenario, calibration procedure and slip effects is shown in the video attachment.

\section{A. Slip Model Calibration}

The goal of the demo mission was the simulation of a realistic planetary exploration scenario. Thus, ground-truth position measurements via Differential GPS (DGPS) were only used for offline data verification during the whole 


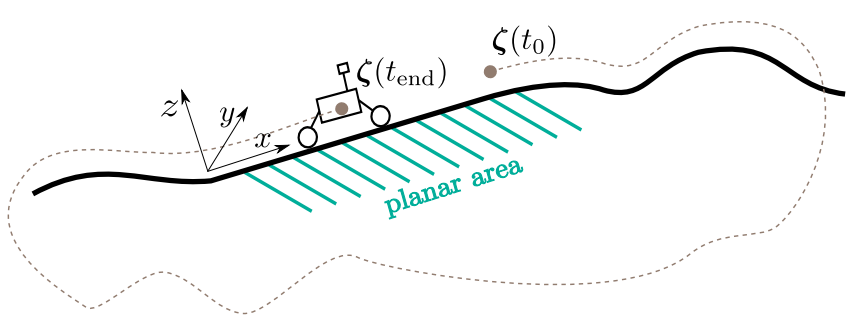

Fig. 4. Experiment setup illustration: LRU1 with constructed coordinate frame on planar, inclined terrain in the vicinity of the frame origin

test campaign. The calibration of the slip parameters was therefore performed using only information that is potentially available after landing on a foreign planet, that is start and end point of a driven calibration trajectory. The calibration experiments were performed in a sector of variable inclination angles spanning over $20 \mathrm{~m}$ in both directions. The region for end-point measurements was chosen to lie within an approx. planar area with a slope of about $5 \mathrm{deg}$, to comply with the $2 \mathrm{D}$ requirement that is posed by the analytical parameter Jacobian matrix computation (see Fig. 4). To allow for the exact measurement of the 2D start and end point positions, a coordinate system was manually created on the test site. To get sufficient data for determining six parameters without overfitting, we chose a set of 19 calibration trajectories shown in Fig. 5. The length of the trajectories was between $5 \mathrm{~m}$ and $40 \mathrm{~m}$. The trajectories were chosen according to the following aspects:

- Different driving modes of the rover should be present. During the demo mission, two locomotion modes were used: In the first mode, only $x$-velocity and yaw rate $\omega$ are commanded, such that $\beta \equiv 0$. In the second mode only $x$-velocity and $y$-velocity are commanded, the desired yaw rate is zero.

- All modeled aspects of slip should appear in the complete calibration dataset (e.g. driving parallel and perpendicular to sloped terrain).

- The shorter trajectories (1) - (15) were chosen such that each trajectory should display mainly one of the modeled slip aspects.

- The longer trajectories (16) to (19) contained all slip effects.

- There should be a set of trajectories without loop closures, as they can distort the calibration result.

Afterwards, the slip parameters were computed according to (9) with an update rate of $\alpha=0.7$ and 5 iterations. The step size was $10 \mathrm{~ms}^{2}$. Due to their length, trajectories (16) to (19) were weighted with the factor 0.5 during calibration.

The results of the calibration can be inspected in Fig. 6. It can be observed that the dominating effects while traversing inclined terrain are slope-dependent slip in longitudinal and lateral direction, described by $p_{1}$ and $p_{3}$ respectively. The constant rolling resistance parameter $p_{2}$ is calibrated to approx. $11 \%$ of the translational velocity. The last three parameters $p_{4}-p_{6}$ describing rotational slippage effects are comparatively small.

\footnotetext{
${ }^{2}$ Test computations with smaller step sizes and more iterations showed no significant improvement of the calibration accuracy.
}
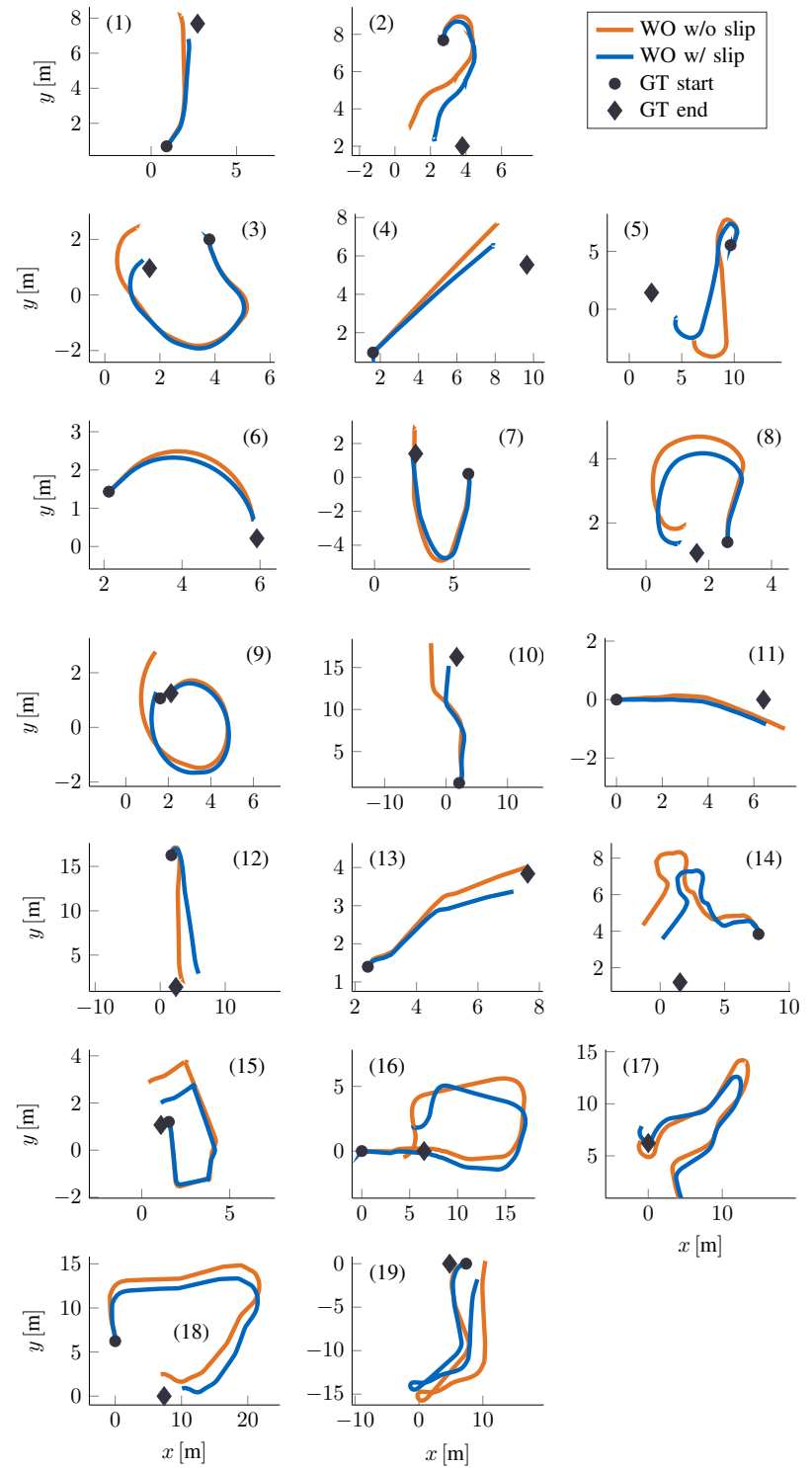

Fig. 5. The set of trajectories used for the calibration of slip parameters Ground truth (GT) start and end points and wheel odometry (WO) before (w/o) and after (w/) calibration are shown.

\begin{tabular}{|c|c|c|c|c|c|}
\hline$p_{1}$ & $p_{2}$ & $p_{3}$ & $p_{4}$ & $p_{5}$ & $p_{6}$ \\
\hline 0.6367 & 0.1089 & 0.2465 & -0.0076 & 0.0061 & 0.0733 \\
\hline
\end{tabular}

Fig. 6. Slip parameters resulting from calibration.

\section{B. Validation During a Long-Range Drive}

The calibrated slip parameters were validated during a teleoperated long range drive of approx. $900 \mathrm{~m}$. The trajectory at the test site can be inspected in Fig. 7. After starting close to the base camp at the position marked with (0), the rover drives on relatively flat terrain next to the calibration area until it reaches (1). Afterwards, it drives downhill for approx. $100 \mathrm{~m}$ (2) until it reaches the lowest point where it traverses horizontally on a flat area (3). The uphill part of the trajectory is denoted by (4). A second circle of the same trajectory is driven (small deviations at (2)). Before returning to the base camp, a third small circle was driven close to (1).

Figure 8 shows the linear part of the wheel velocity 


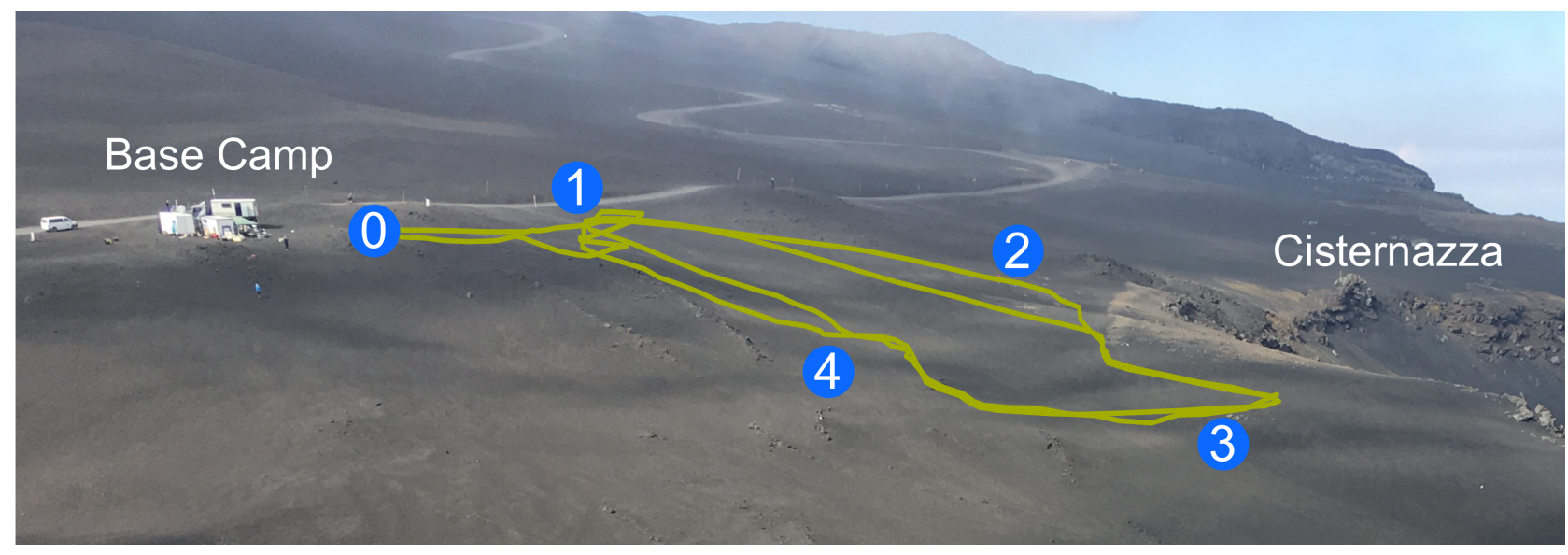

Fig. 7. Aerial view of the test site with the DGPS trajectory of the long-range drive. The overall length of the trajectory is approx. $900 \mathrm{~m}$.

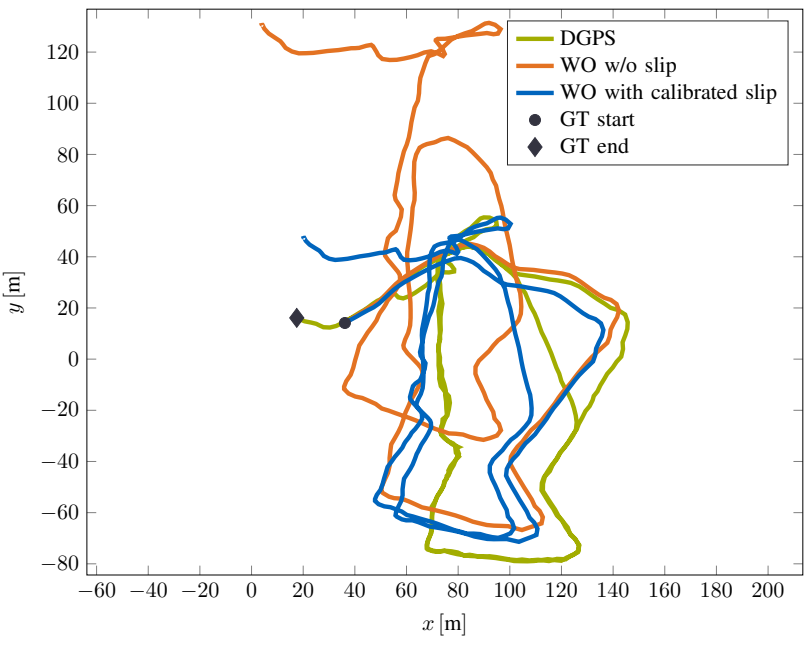

Fig. 8. Integrated wheel odometry (WO) velocity measurements during the long-range test, compared with the DGPS data. Only the linear velocities are used from the wheel odometry, angular velocities are from the EKF output.

measurements. For the sake of clarity, they are plotted with the rotation taken from the output of the extended Kalman Filter used for pose estimation. As only linear velocities are fused into the EKF (cf. Fig. 9), this shows the improvement of the WO measurements that are relevant for pose estimation and thus for the autonomous exploration scenario represented by the long range drive. It can be seen that without slip estimation, the driven distance is significantly over-estimated, especially during the uphill parts of the trajectory (4). In comparison, the slip-aware WO delivers a continuous slight under-estimation of the driven distance over all parts of the trajectory. Due to the fact that the rolling resistance is slope independent, this leads to the hypothesis that parameter $p_{2}$ was estimated too high during the calibration. We trace this back to the fact that the measurement site for the calibration trajectories was frequently passed by persons. This further loosened the soil which increased the rolling resistance specifically in this area and caused more wheel slippage. Nevertheless, it can be observed from Fig. 8 that the effects of slope-dependent slip are almost non-existent and thus it is assumed that the values of $p_{1}$ and $p_{3}$ are sufficiently

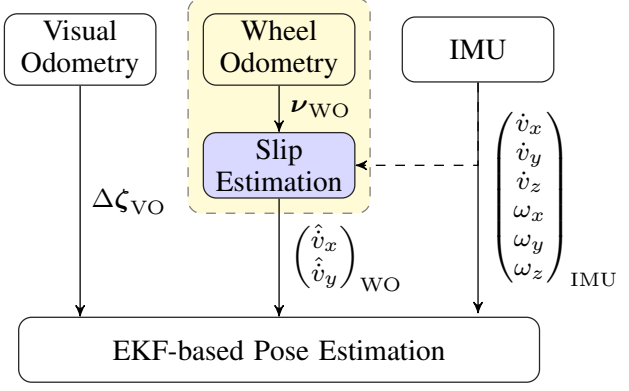

Fig. 9. Filter architecture for pose estimation. Only linear velocities in $x$ and $y$-direction are used from wheel odometry. The implementation of the EKF is based on the concept detailed in [7].

exact. As result of these findings, the WO computations were replicated with a manually decreased parameter $p_{2}=0.036$ and the other parameters as indicated in Fig. 6. The result can be inspected in Fig. 10, where the accumulated distance error is plotted for different parameter sets. During the two uphill drives (starting approximately after $270 \mathrm{~m}$ and $610 \mathrm{~m}$, marked in the plot), the wheel odometry without slip consideration clearly overestimates the driven distance while it underestimates the distance during the downhill parts due to skidding. Using the calibrated slip parameters eliminates the slope-dependent effects and only a constant relative error remains - which is then removed by adapting $p_{2}$.

In Fig. 11 the relative error, defined as the ratio of the errors between the estimated and true length of the segment to the true segment length, is depicted for the different approaches. It can be seen that the calibrated wheel odometry already reduces the dispersion of the relative error significantly, while shifting the median error to approx. $-8 \%$. This again indicates that the error is introduced mainly by parameter $p_{2}$. After manually tuning $p_{2}$, the median of the relative error is below $1 \%$ and $90 \%$ of the data lie within $\pm 5 \%$ which validates the assumption.

Of course, atypical soil properties might also be present at calibration areas on foreign planets. Updating the (precalibrated) parameters continuously while driving seems to be one possibility to overcome this problem. To validate this method, the constant slip parameters $\boldsymbol{p}$ are compared with a 


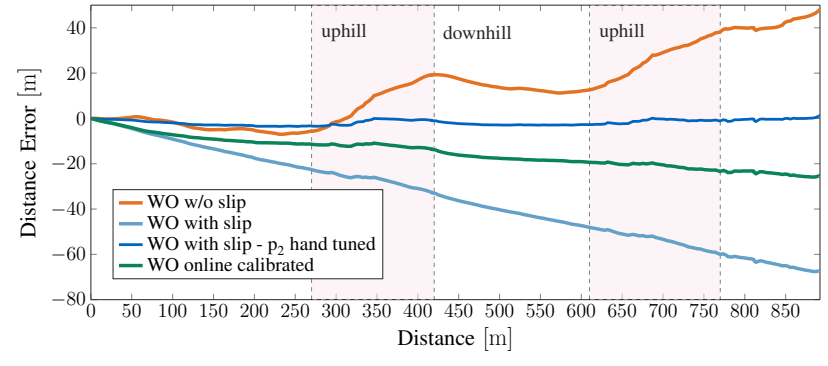

Fig. 10. The offset between the estimated distance of the wheel odometry (WO) and the DGPS data during the long-range test, considering parameters from offline and online estimation methods. Uphill passages are marked in magenta.

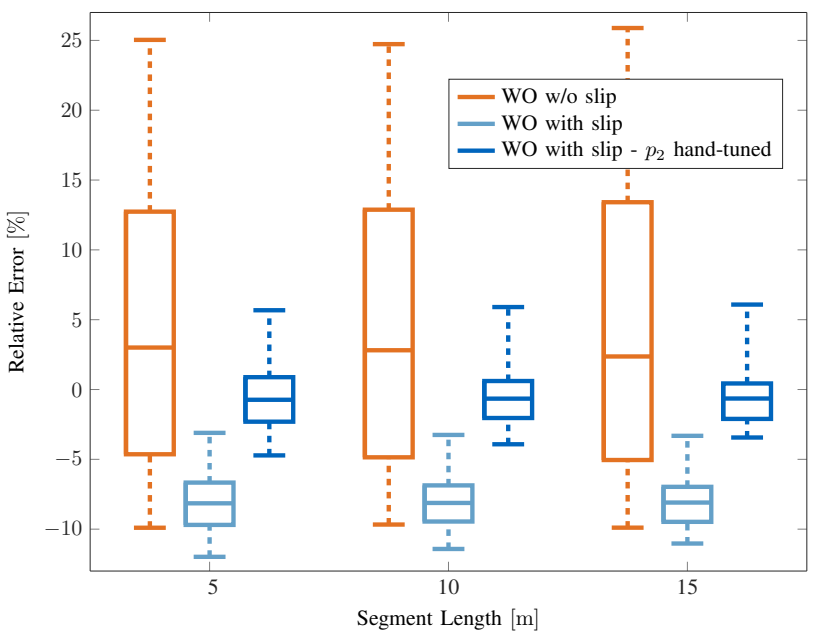

Fig. 11. Comparison of the relative error for the different approaches and segment lengths of 5,10 and $15 \mathrm{~m}$.

set of parameters calibrated online during the long range drive. Therefore, the online parameter estimation method proposed by [15] is applied to the data of the long range test, employing DGPS data as ground truth. An EKF is used to estimate the parameters using piece-wise sections of the trajectory of approx. $2 \mathrm{~m}$ length. The parameters from Fig. 6 are used as start values and the change of parameters over the trajectory is shown in Fig. 12. It can be seen that all parameters except $p_{2}$ exhibit close to constant behavior, confirming the parameters obtained by the offline calibration. As described in the previous section, we assumed $p_{2}$ to be estimated too high due to loose soil specifically at the calibration site. This assumption is supported by the online parameter estimation, as the value of $p_{2}$ decreases significantly over the trajectory. At approximately $400 \mathrm{~m}$ of the drive - close to (1) - the rover crossed the calibration site, causing the local rise of the parameter $p_{2}$. Note that a loss in DGPS precision is negatively affecting the parameter estimation during the last $150 \mathrm{~m}$ of the trajectory. The accumulated distance error of the online calibrated WO can also be inspected in Fig. 10. Due to the high initial value of $p_{2}$ and the slow convergence to lower values as a consequence of parameterization of the online estimation, the resulting distance is still under-estimated. Nevertheless, it can be observed that online calibration can cope with erroneous calibration results or changing terrain properties

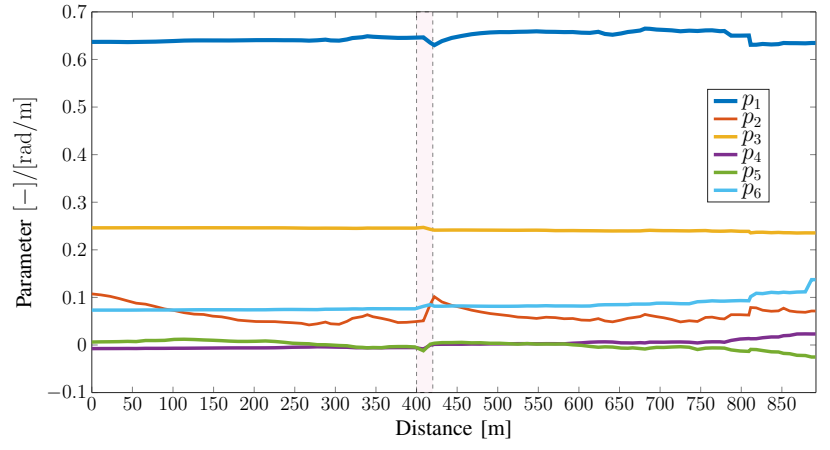

Fig. 12. Slip parameters as result of the simulated online parameter estimation using DGPS as ground truth information. Start values are the calibrated values (see Fig. 6). The part of the trajectory passing the calibration area is marked in magenta. Note that the loss in DGPS precision is negatively affecting the parameter estimation during the last $150 \mathrm{~m}$ of the trajectory.

while driving. Note that the mentioned online calibration was performed to verify the method during post-processing and thus utilized DGPS data. In real space exploration scenarios, reference data must be obtained from other sources, which will be discussed in Sec. IV.

\section{DISCUSSION}

As shown in Sec. III, the offline calibrated slip model led to a significant improvement of the wheel odometry measurements. Particularly the fact that no ground-truth measurements besides known start and end point of the calibration trajectories are needed, makes the approach applicable for a potential use in planetary exploration. A possible scenario could be the autonomous calibration after landing on a foreign planet by reference to visual markers brought along on the lander and expressive landmarks close to the landing site, providing constant slip parameters for later exploration in distant areas.

However, the presented results show also room for improvements. The results presented in Sec. III-B suggest that the assumption of homogeneous soil properties does not hold unrestrictedly. Therefore, online slip parameter updating could improve the performance significantly. In the absence of ground truth position measurements, methods like proposed in [13] or [21] could be used, where wheel odometry is compared and corrected w. r. t. a reference signal from fusing only IMU and VO measurements. This can be used for estimating slip in applications like traction control, but not in the sensor fusion for pose estimation (to avoid correlations). If one of the reference sensors fails, WO can be used as backup sensor in the pose estimation for that case.

An interesting observation from the results is that there might be non-linear correlations between body velocity and slip that the used slip model (15) cannot describe. This is not explicitly discussed in Sec. III for reasons of conciseness. Nevertheless, an investigation of different slip models or even non model-dependent approaches such as learning could be beneficial and will be a topic of future work. In our opinion, especially machine learning has strong potential, as we observed the slip to depend on many additional factors. One example are oscillations of the body introduced by 
mechanic elasticities, which are excited by the wheel-soil interaction and are complex to model analytically.

Another point is that the knowledge of the wheel-soil contact angle could further improve the results of wheel odometry measurements, especially for pitch and roll movements of the rover. So far, the assumption that the contact point is always the vertical projection of the wheel hub to the ground w.r.t. the wheel frame is causing systematic errors especially on sloped terrain, as the vertical velocity component of the wheel is neglected and the x-component is over-estimated. A robust estimation based on the available sensor readings is a problem not yet solved in our setup. Approaches from literature to estimate the contact angles from wheel speeds and pitch rate assume no slip (e.g. [22]), while in other approaches, additional sensors are required (e. g. [23], [24]).

Finally, we want to highlight that the results shown in this paper vary significantly from the experiments presented in [15] and [16]. Seegmiller et. al. showed mainly experiments with skid-steered vehicles, where rotational slip effects are much more significant. Also, the vehicle soil interaction properties are different for the large, stiff vehicles they use on paved terrain, grass and muddy soils. Last, we embed our results in the context of an autonomous planetary exploration mission with all restrictions that come along with it, while the results available in the mentioned literature utilize high-end sensors for ground truth measurement and a great amount of knowledge on the terrain (e.g. slope and wheel contact angle). Still, we validate that the methods proposed by [15] are applicable even in extreme environments and realistic space exploration scenarios, and lead to a significant improvement while taking low effort both in experiment design and in computational complexity.

\section{CONCLUSION}

In this paper, we showed recent results of slip modeling and estimation in the context of a space-analogue test campaign on Mt. Etna. A model-based approach for body slip estimation was adopted and implemented on a fourwheeled rover system. Results both from the calibration in a delimited area and from the validation in a long range drive of approx. $900 \mathrm{~m}$ were presented. It was shown that the presented approach for slip estimation leads to a significant improvement of the resulting wheel odometry measurements. Nevertheless, the results imply that the approach could not describe all occurring effects of the wheel-soil interaction observed on volcanic terrain. The results were discussed w. r.t. the advantages of the method but also possible improvements were pointed out.

\section{REFERENCES}

[1] A. H. Mishkin, J. C. Morrison, T. T. Nguyen, H. W. Stone, B. K. Cooper, and B. H. Wilcox, "Experiences with operations and autonomy of the mars pathfinder microrover," in 1998 IEEE Aerospace Conference Proceedings, vol. 2, March 1998, pp. 337-351.

[2] W. Gong, "Discussions on localization capabilities of MSL and MER rovers," Annals of GIS, vol. 21, no. 1, pp. 69-79, 2015.

[3] M. J. Schuster, C. Brand, S. G. Brunner et al., "The LRU Rover for Autonomous Planetary Exploration and its Success in the SpaceBotCamp Challenge," in 2016 IEEE International Conference on Autonomous Robot Systems and Competitions (ICARSC), May 2016.
[4] R. Haarmann, Q. Mühlbauer, L. Richter et al., "Mobile Payload Element (MPE): concept study of a small, outonomous and innovative sample fetching rover," in Proc. of the 12th Symposium on Advanced Space Technologies in Robotics and Automation (ASTRA), 2013.

[5] M. J. Schuster, S. G. Brunner, K. Bussmann et al., "Towards Autonomous Planetary Exploration: The Lightweight Rover Unit (LRU), its Success in the SpaceBotCamp Challenge, and Beyond," Journal of Intelligent \& Robotic Systems (JINT), Nov. 2017.

[6] M. Bajracharya, M. W. Maimone, and D. Helmick, "Autonomy for mars rovers: Past, present, and future," Computer, vol. 41, no. 12, pp. 44-50, Dec 2008.

[7] K. Schmid, F. Ruess, M. Suppa, and D. Burschka, "State estimation for highly dynamic flying systems using key frame odometry with varying time delays," in Proc. of the 2012 IEEE/RSJ International Conference on Intelligent Robots and Systems, 10 2012, pp. 2997-3004.

[8] K. Schmid, F. Ruess, and D. Burschka, "Local reference filter for lifelong vision aided inertial navigation," in 17th International Conference on Information Fusion (FUSION), July 2014, pp. 1-8.

[9] Alfred-Wegener-Institute - Helmholtz-Center for Polar- and Marine Research, "Robex - Robotic Exploration of Extreme Environments," Webpage, accessed 15 July 2017. [Online]. Available: http://www.robex-allianz.de/

[10] A. Wedler, M. Vayugundla, H. Lehner et al., "First Results of the ROBEX Analogue Mission Campaign: Robotic Deployment of Seismic Networks for Future Lunar Missions," in 68th International Astronautical Congress (IAC). Adelaide, Australia: International Astronautical Federation (IAF), Sept. 2017.

[11] A. Wedler, M. Hellerer, B. Rebele et al., "Robex - components and methods for the planetary exploration demonstration mission," in 13th Symposium on Advanced Space Technologies in Robotics and Automation (ASTRA). ESA Website, 2015.

[12] D. Liang, D. Zongquan, G. Haibo, T. Jianguo, I. K. D., and L. Guangjun, "Interaction mechanics model for rigid driving wheels of planetary rovers moving on sandy terrain with consideration of multiple physical effects," Journal of Field Robotics, vol. 32, no. 6, pp. 827-859.

[13] D. Helmick, Y. Cheng, D. Clouse, M. Bajracharya, L. Matthies, and S. Roumeliotis, "Slip compensation for a Mars Rover," in Proc. of the 2005 IEEE/RSJ International Conference on Intelligent Robots and Systems (IROS), September 2005, pp. 2806 - 2813.

[14] L. Ojeda, D. Cruz, G. Reina, and J. Borenstein, "Current-based slippage detection and odometry correction for mobile robots and planetary rovers," IEEE Transactions on Robotics, vol. 22, no. 2, pp. 366-378, April 2006.

[15] N. Seegmiller, F. Rogers-Marcovitz, G. A. Miller, and A. Kelly, "Vehicle model identification by integrated prediction error minimization," International Journal of Robotics Research, vol. 32, no. 8, pp. 912931, July 2013.

[16] N. Seegmiller and A. Kelly, "Enhanced 3D Kinematic Modeling of Wheeled Mobile Robots," in Proc. of Robotics: Science and Systems $X, 2014$.

[17] A. Kelly, "Linearized error propagation in odometry," International Journal of Robotics Research, vol. 23, pp. 179-218, 2004.

[18] M. Bekker, Introduction to Terrain-Vehicle Systems. Univ. of Michigan Press, 1969.

[19] L. Preston, M. Grady, and S. Barber, "TN2: The Catalogue of Planetary Analogues," The Planetary and Space Sciences Research Institute, The Open University, UK, Dec. 2012, under ESA contract: 4000104716/11/NL/AF.

[20] G. Lanzafame, M. Neri, V. Acocella, A. Billi, R. Funiciello, and G. Giordano, "Structural features of the July-August 2001 Mount Etna eruption: evidence for a complex magma supply system," Journal of the Geological Society, vol. 160, pp. 531-544, January 2003.

[21] D. M. Helmick, S. I. Roumeliotis, Y. Cheng, D. S. Clouse, M. Bajracharya, and L. H. Matthies, "Slip-compensated path following for planetary exploration rovers," Advanced Robotics, vol. 20, no. 11, pp. 1257-1280, 2006.

[22] K. Iagnemma and S. Dubowsky, "Vehicle wheel-ground contact angle estimation: with application to mobile robot traction control," in Advances in robot kinematics. Springer, 2000, pp. 137-146.

[23] P. Lamon, "3D-Position Tracking and Control for All-Terrain Robots," $\mathrm{Ph} . \mathrm{D}$. dissertation, École polytechnique fédérale de Lausanne, 2005.

[24] H. Xu, X. Liu, H. Fu, B. B. Putra, and L. He, "Visual Contact Angle Estimation and Traction Control for Mobile Robot in Rough-Terrain," Journal of Intelligent \& Robotic Systems, vol. 74, no. 3, pp. 985-997, June 2014. 